Collection SFN 9 (2008) 111-119

(C) EDP Sciences, Les Ulis

DOI: $10.1051 / \mathrm{sfn}: 2008008$

\title{
Complémentarité de la diffraction de neutrons avec d'autres techniques spectroscopiques (dans l'étude du magnétisme)
}

\author{
P. Bonville \\ CEA, Centre de Saclay, DSM/Service de Physique de l'État Condensé, \\ 91191 Gif-sur-Yvette, France
}

\begin{abstract}
La complémentarité entre les mesures de diffraction de neutrons et les informations obtenues par trois techniques spectroscopiques locales (spectroscopie Mössbauer, spectroscopie de muons et résonance magnétique nucléaire) dans l'étude du magnétisme est examinée à l'aide de quelques exemples. Les principes de spectrométrie de ces diverses techniques sont exposés, et les spécificités des mesures locales sont soulignées.
\end{abstract}

\section{INTRODUCTION}

Nous allons nous intéresser ici à trois techniques spectroscopiques qui sont très utilisées dans les études de magnétisme électronique et qui fournissent des informations soit originales, soit complémentaires de celles obtenues par la diffraction de neutrons : la spectroscopie Mössbauer d'absorption (SM), la spectroscopie de muons positifs ( $\mu \mathrm{SR})$ et la résonance magnétique nucléaire (RMN). Cette dernière technique, bien sûr, possède un très large domaine d'applications en dehors du magnétisme, dont nous ne parlerons pas. Ces trois techniques utilisent le couplage des spins électroniques soit avec le spin nucléaire de l'atome magnétique (interaction hyperfine directe : SM, RMN parfois), soit avec celui d'un autre atome (interaction hyperfine transférée : RMN dans la majorité des cas), soit avec le spin d'une particule-sonde, le muon positif $\left(\mu^{+}\right)$, implantée dans l'échantillon (interaction dipolaire et hyperfine : $\mu \mathrm{SR})$. C'est une différence importante avec la diffraction de neutrons, où le spin du neutron interagit directement avec le spin électronique. En outre, ces trois techniques spectroscopiques sont peu sensibles à l'arrangement spatial des moments magnétiques dans la phase ordonnée et peuvent être considérées comme des mesures locales. Cependant, en spectroscopie Mössbauer et, dans une moindre mesure en RMN, le spectre peut présenter une forme particuliére dans certains cas de structures magnétiques exotiques, comme les spirales, les structures modulées incommensurables, ...

Il convient de garder à l'esprit une différence importante : la diffraction magnétique des neutrons ne peut donner accés qu'à la composante ordonnée du moment magnétique, alors que les techniques spectroscopiques locales sont sensibles au module du moment. Quant à la dynamique des moments électroniques, elle peut être approchée par la diffusion inélastique des neutrons et par les trois techniques locales dont nous allons parler : on peut mesurer ainsi les lois de dispersion des magnons, ou bien les fluctuations (souvent à caractère quantique) des moments individuels dans la phase d'ordre magnétique. La gamme de fréquences accessibles est assez large (en gros de quelques $\mathrm{MHz}$ à quelques $\mathrm{THz}$ ) et dépend de la technique particuliére. La dynamique des moments magnétiques est un domaine très intéressant, mais vaste, et qui sort du domaine de cet exposé.

Quel type de rayonnement détecte-t-on dans les trois techniques citées? La SM et la RMN sont des spectroscopies d'absorption de photons, bien que d'un caractére très différent dans chaque cas. La SM utilise la transition entre le niveau fondamental nucléaire et le premier niveau excité, dont l'énergie doit être comprise entre 10 et $100 \mathrm{keV}$ pour que ce genre de mesure soit possible. Les photons absorbés sont donc des photons $\gamma$, issus de la décroissance radioactive d'un noyau parent, dans la spectroscopie classique (ou "historique"). Depuis 1995, les photons X mous du rayonnement synchrotron, dans la même gamme d'énergie, permettent de mettre en oeuvre une forme particulière de spectroscopie 
Mössbauer. En RMN, la transition utile est celle qui sépare les niveaux Zeeman de l'état fondamental nucléaire, en présence d'un champ magnétique externe dans la grande majorité des cas. Les photons concernés sont des photons micro-onde, car les écarts Zeeman nucléaires sont de l'ordre de $10^{-7} \mathrm{eV}$, correspondant à des fréquences de quelques $10 \mathrm{MHz}$. Par contre, en $\mu \mathrm{SR}$, on détecte les positrons $e^{+}$ émis lors de la décroissance par interaction faible du $\mu^{+}$, qui a un temps de vie $\tau_{\mu}=2.2 \mu \mathrm{s}$. La direction préférentielle suivant laquelle les positrons sont émis est la direction qu'avait le spin $S_{\mu}=1 / 2$ du muon à l'instant de la décroissance. Comme le spin du muon précesse dans le champ dipolaire dû aux moments électroniques (et aussi nucléaires) présents dans l'échantillon, le taux de comptage des positrons en fonction du temps permet d'obtenir des informations sur ces moments.

Nous allons passer en revue ces trois techniques, en décrivant leur principe et en donnant des éléments de spectrométrie, puis en illustrant par des exemples la spécificité ou la complémentarité des informations qu'elles fournissent.

\section{SPECTROSCOPIE MÖSSBAUER D'ABSORPTION}

La spectroscopie Mössbauer, ou absorption résonante nucléaire de photons, est rendue possible par la découverte en 1958 par R. Mössbauer de l'effet qui porte son nom. Mössbauer a montré que l'absorption résonante de photons entre niveaux nucléaires, si elle est quasiment nulle dans un milieu gazeux ou liquide à cause de la disparité entre l'énergie de recul du noyau et la faible largeur de la raie, devient par contre possible dans un solide où les atomes (et donc les noyaux) sont liés entre eux par les liaisons chimiques. Plus précisément, la probabilité d'absorption/émission sans recul (ou plus justement sans excitation ou absorption de phonons) d'un photon par un noyau dans un solide est finie à température nulle, et décroît lorsque la température augmente. À température nulle, cette probabilité, ou facteur-f, vaut :

$$
f(T)=\exp \left(-\frac{-3 E_{0}^{2}}{4 M c^{2} k_{B} \Theta_{D}}\right),
$$

où $E_{0}$ est l'énergie de la transition nucléaire, $M$ la masse du noyau et $\theta_{D}$ la température de Debye du réseau cristallin. L'effet Mössbauer n'existe que pour un nombre limité d'isotopes ; le plus couramment utilisé est le ${ }^{57} \mathrm{Fe}\left(E_{0}=14.4 \mathrm{keV}\right)$. Parmi les terres rares, on peut citer ${ }^{170} \mathrm{Yb}\left(E_{0}=84.3 \mathrm{keV}\right)$ et ${ }^{155} \mathrm{Gd}$ $\left(E_{0}=86.5 \mathrm{keV}\right)$. Les caractéristiques des niveaux nucléaires de ${ }^{170} \mathrm{Yb}$, qui nous servira d'exemple dans la suite, sont les suivantes : spin de état fondamental $I_{f}=0$, spin du premier niveau excité $I_{e}=2$, demi-vie de ce niveau métastable : $\tau_{n}=1.59 \mathrm{~ns}$, ce qui lui confère une largeur en énergie $\Gamma_{n}=2.87 \times 10^{-7} \mathrm{eV}$. Les interactions hyperfines entre le moment électronique et le spin du noyau étant de l'ordre de $10^{-6} \mathrm{eV}$ (par exemple, les écarts Zeeman nucléaires en présence d'un champ magnétique hyperfin), on voit que la faible largeur de la raie Mössbauer permettra de les résoudre. La spectroscopie Mössbauer est donc une spectroscopie hyperfine qui permet de mesurer les champs internes au matériau, même en l'absence de champ magnétique extérieur [1].

Nous allons décrire briévement le principe de la spectrométrie Mössbauer d'absorption en géométrie de transmission (voir Fig. 1) : la source (S) émet des photons $\gamma$ qui traversent l'échantillon à étudier (1'“absorbeur" A) et qui sont détectés dans le compteur (C) s'ils n'ont pas été absorbés auparavant entre les niveaux nucléaires de l'absorbeur. Le balayage en énergie des photons nécessaire à l'obtention du spectre d'absorption est effectué par effet Doppler en montant la source sur une tige vibrante (T), dont la vitesse est asservie $(\mathrm{SV})$ à une fonction périodique $(\mathrm{F}$, en général linéaire avec le temps). L'énergie des photons est donc : $E(v)=E_{0}(1+v / c)$. Grâce à une électronique adéquate de synchronisation du mouvement et du comptage, on obtient des spectres en énergie $\left(\mathrm{N}_{i}, \mathrm{v}_{i} \propto E_{i}\right)$. La source est en général une matrice métallique contenant sous forme diluée le parent radioactif de l'isotope considéré (le ${ }^{170} \mathrm{Tm}^{*}$ pour ${ }^{170} \mathrm{Yb}$ ). Dans les installations auprés d'un synchrotron, la spectrométrie est différente : les photons incidents sont des $\mathrm{X}$ mous à dispersion en énergie suffisante pour exciter toutes les transitions nucléaires, et on observe en fonction du temps les battements de photons lors de la décroissance radioactive. 


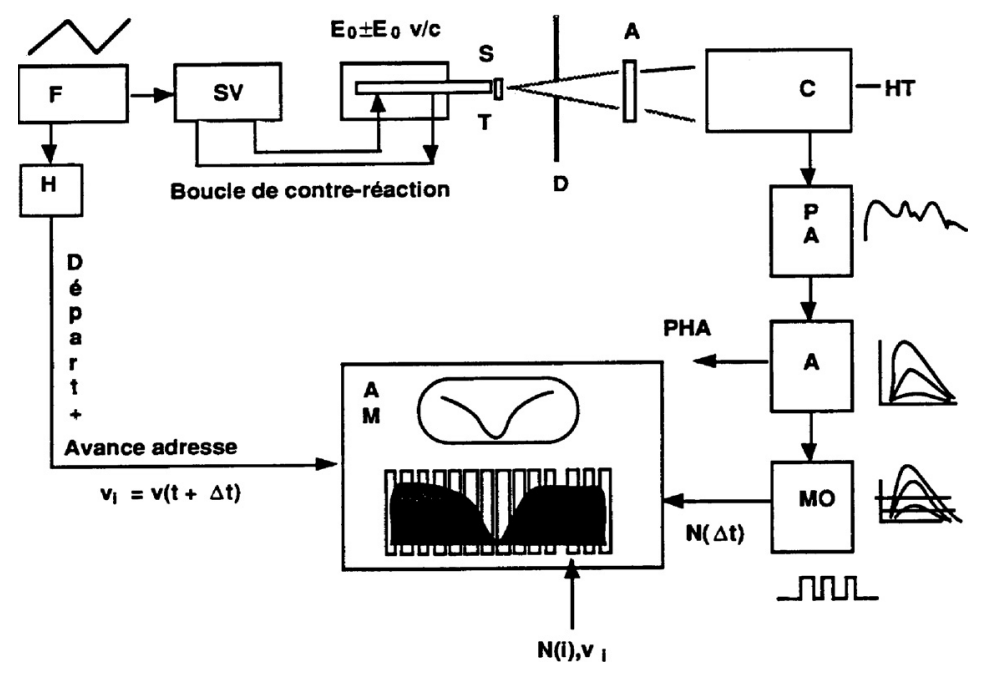

Figure 1. Schéma d'un spectromètre Mössbauer en géométrie de transmission.

Dans un matériau ferro-, ferri-ou antiferromagnétique, les couches électroniques polarisées créent un champ magnétique hyperfin $\mathbf{H}_{h f}$ au site du noyau; la dégénérescence du multiplet de spin nucléaire $I$ est donc levèe par l'effet Zeeman (hyperfin), conduisant à de faibles écarts en énergie de l'ordre de $10^{-6}-10^{-7} \mathrm{eV}$. L'interaction magnétique hyperfine provient de deux mécanismes principaux :

1. le mécanisme de "contact" dû aux électrons de type $s(l=0)$ qui peuvent pénétrer à l'intérieur du noyau. L'interaction s'écrit : $\mathcal{H}_{c}=A_{c} \mathbf{I} \cdot\langle\mathbf{S}\rangle$, où $A_{c}$ est la constante hyperfine de contact et $\mathbf{S}$ le spin électronique.

2. le mouvement orbital des électrons, qui donne lieu à un terme du même type : $\mathcal{H}_{L}=\alpha_{L} \mathbf{I} \cdot\langle\mathbf{L}\rangle$ pour les ions de transition $3 d-4 d$, $\mathbf{L}$ étant le moment orbital, et : $\mathcal{H}_{J}=A_{J} \mathbf{I} \cdot\langle\mathbf{J}\rangle$ pour les terres rares, J étant le moment angulaire total.

En écrivant l'interaction hyperfine sous la forme du produit scalaire du moment magnétique nucléaire $g_{n} \mu_{n} \mathbf{I}$, où $\mu_{n}$ est le magnéton de Bohr nucléaire, et du champ hyperfin, on obtient aisément l'expression de ce dernier (dans le cas des terres rares) :

$$
H_{h f}=\frac{\left(g_{J}-1\right) A_{c}+A_{J}}{g_{n} g_{J} \mu_{n} \mu_{B}} \mu,
$$

$\boldsymbol{\mu}=g_{J} \mu_{B}\langle\mathbf{J}\rangle$ étant le moment électronique. La proportionalité du champ hyperfin et du moment (qui est aussi valable dans le cas de $\mathrm{Fe}^{3+}$ ) montre tout l'intérêt de la spectroscopie Mössbauer dans l'étude du magnétisme : en suivant la variation thermique (par exemple) de $H_{h f}$, on obtient celle du moment électronique. Quant aux ordres de grandeur, les champs hyperfins dans les composés de terre rare peuvent atteindre quelques centaines de $\mathrm{T}$, et dans les composés $3 d-4 d$ quelques dizaines de $\mathrm{T}$.

À titre d'exemple, je présenterai l'étude de la transition magnétique dans YbAs, un composé cubique où l'ion $\mathrm{Yb}$ est trivalent paramagnétique. Les spectres Mössbauer sur l'isotope ${ }^{170} \mathrm{Yb}$ sont représentés sur la Figure 2 à gauche, à très basse température. A $0.05 \mathrm{~K}$, on observe les 5 raies attendues en présence d'un champ hyperfin, qui laisse inchangé le niveau fondamental nucléaire $\left(I_{f}=0\right)$, mais qui scinde en 5 états le niveau excité $\left(I_{e}=2\right)$. Pour l'isotope ${ }^{170} \mathrm{Yb}$, les 5 transitions nucléaires ont la même intensité dans le cas d'une poudre, ce qui donne 5 raies d'absorption d'égale hauteur. La présence du champ hyperfin est le signe d'un ordre magnétique des moments des ions $\mathrm{Yb}^{3+}$. A $0.65 \mathrm{~K}$, on n'observe plus qu'une seule raie, indice de l'annulation du champ hyperfin, et donc du passage en phase paramagnétique à $T_{N} \simeq 0.65 \mathrm{~K}$. Mais on observe que la raie unique apparaît en-dessous de $T_{N}$ : par exemple, on la voit nettement à $0.55 \mathrm{~K}$. En outre, à mesure que la température augmente, on constate 



Figure 2. À gauche : spectres Mössbauer sur ${ }^{170} \mathrm{Yb}$ dans $\mathrm{YbAs}$; ̀̀ droite : variation thermique du champ hyperfin (a) et de la fraction ordonnée magnétiquement (b) d'après la Référence [2].

que l'écart entre les raies extrêmes du spectre, qui donne la mesure du champ hyperfin, varie très peu. Ces deux phénomènes, l'absence de variation thermique de $H_{h f}$, donc du moment magnétique, et la coexistence dans l'échantillon prés de $T_{N}$ de régions ordonnées et paramagnétiques, sont la marque d'une transition du premier ordre (voir Figure 2 à droite).

Des mesures de diffraction de neutrons à très basse température furent réalisées dans YbAs peu après l'étude Mössbauer [3]. La structure magnétique fut déterminée comme un ordre antiferromagnétique de type III, avec un vecteur de propagation $\mathbf{k}=\left(1,0, \pm \frac{1}{2}\right)$ (voir Figure 3 à gauche), avec une température d'ordre $T_{N} \simeq 0.6 \mathrm{~K}$, en bon accord avec les données Mössbauer. L'intensité de la raie de Bragg (1/2, 0 , 1), proportionnelle au carré du moment ordonné, mais aussi à la fraction du composé où se manifeste l'ordre magnétique, montre une variation thermique (voir Figure 3 à droite) proche, non pas de celle du moment (ou champ hyperfin), mais de celle de la fraction magnétique mesurée sur les spectres Mössbauer (voir Figure 2 à droite b). Dans un cas comme celui-ci, où la zone de coexistence des deux phases autour de $T_{N}$ est relativement étendue, on voit que la diffraction de neutrons seule n'a aucun moyen de déceler la transition du premier ordre. La variation thermique anormale de l'intensité de la raie de Bragg peut être interprétée comme celle du moment magnétique, lors d'une transition du second ordre non-standard, qui ferait intervenir, par exemple, le couplage des quadrupoles $4 f$ [3]. Sur cet exemple, on discerne bien la complémentarité des deux techniques : la structure magnétique est déterminée à l'aide des neutrons, mais c'est la mesure locale de la grandeur du moment (et la mise en évidence microscopique de la coexistence de phases) par spectroscopie Mössbauer qui démontre le caractère premier ordre de la transition. 


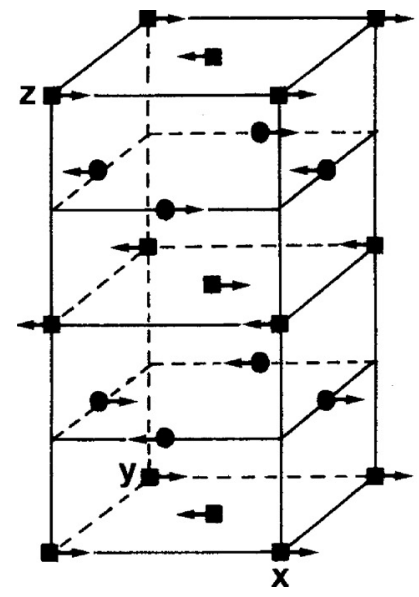

b) YbAs $(0.5,0.0,1.0)$

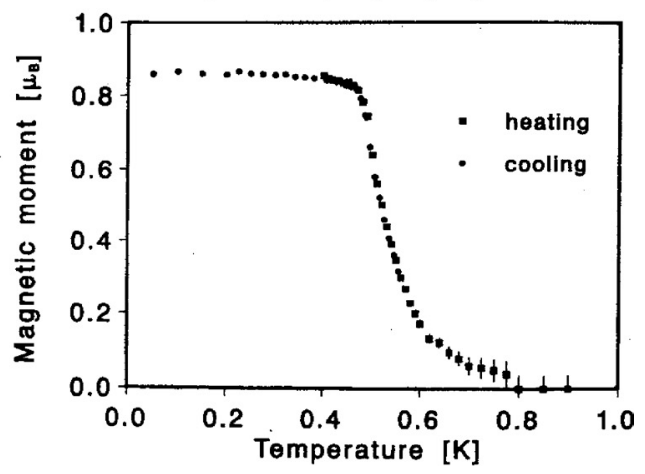

Figure 3. À gauche : structure antiferromagnétique de type III dans YbAs ; les fléches représentent les directions des moments magnétiques ; À droite : variation thermique de l'intensité de la raie de Bragg $(1 / 2,0,1)$, interprétée comme étant celle du moment magnétique de $\mathrm{Yb}^{3+}$, d'après la Référence [3].

\section{LA SPECTROSCOPIE DE MUONS}

La spectrocopie de muons s'est développée à partir des années 1980 auprés des grands instruments (source de neutrons) [4]. Le muon positif $\mu^{+}$est produit par désintégration des pions créés lors du bombardement d'une cible de graphite ou beryllium par un faisceau de protons. Le $\mu^{+}$est un lepton "lourd" instable, de spin $S_{\mu}=1 / 2$, de masse $0.11 \mathrm{~m}_{p}$, où $\mathrm{m}_{p}$ est la masse du proton. Son moment magnétique est donc $\mu_{\mu}=4.82 \times 10^{-3} \mu_{B}$ : il est du même ordre que les moments nucléaires. Son temps de vie est $\tau_{\mu}=2.2 \mu \mathrm{s}$. Lors de leur production, les muons ont leur impulsion directement opposée au spin (Figure 4 à gauche) ; ceci implique qu'un faisceau de muons est pratiquement $100 \%$ polarisé en spin, ce qui est un trait essentiel pour la spectroscopie. La désintégration du muon se fait selon la réaction : $\mu^{+} \rightarrow e^{+}+\bar{v}_{e}+v_{\mu}$. La probabilité d'émission du positron $e^{+}$est maximale le long de la direction du spin du muon, ce qui est une conséquence de la violation de la parité dans la désintégration par interaction faible (voir Figure 4 à droite) : $P(\theta)=1+a \cos \theta$, où le paramétre $a$ dépend de l'énergie des muons. La technique consiste à envoyer le faisceau de muons sur l'échantillon, où le $\mu^{+}$s'implante en site intersticiel. En présence de champ magnétique $H$, soit interne, soit appliqué, le
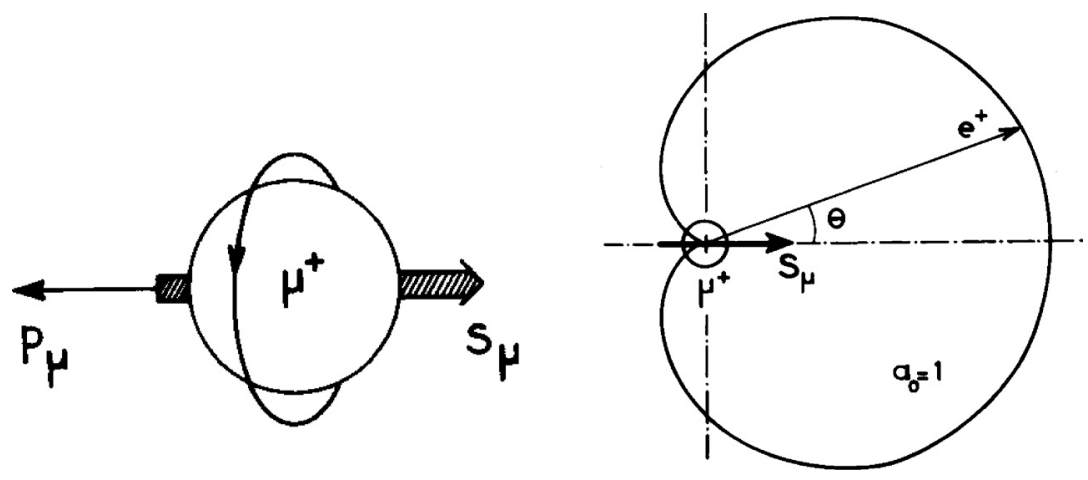

Figure 4. À gauche : impulsion et spin du $\mu^{+}$au moment de sa formation ; À droite : probabilité d'émission du positron dans l'espace lors de la désintégration $\mathrm{du} \mu^{+}$(d'après la Référence [4]). 


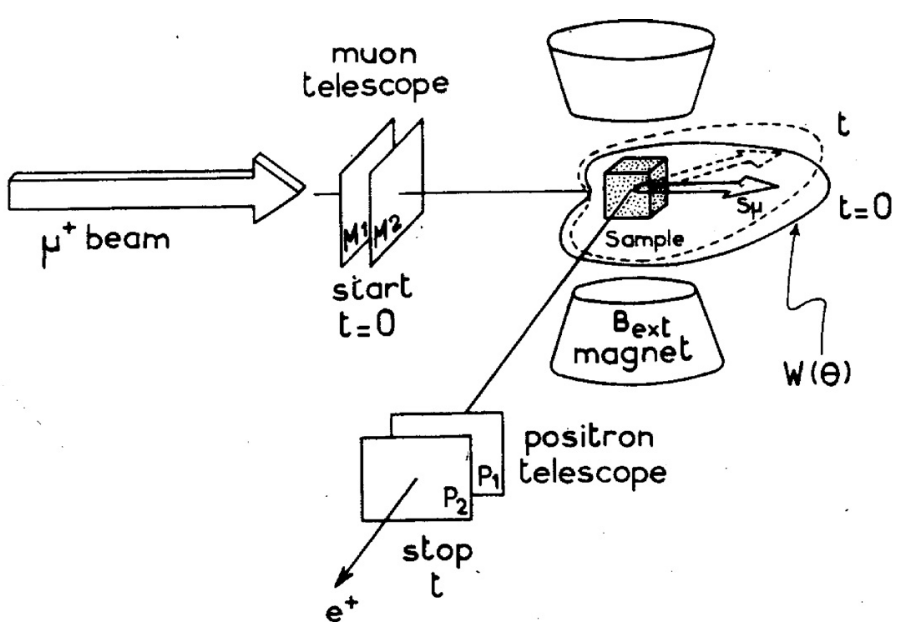

Figure 5. Schéma d'un spectromètre de $\mu \mathrm{SR}$ en géométrie de champ transverse (d'après la Référence [4]).

spin du muon précesse jusqu'à sa désintégration. On détecte le nombre de positrons émis, qui est alors une fonction périodique du temps (superposée à la décroissance exponentielle due au temps de vie) : $N(t)=N_{0} \exp \left(-t / \tau_{\mu}\right)\left[1+a \cos \left(\omega_{\mu}+\Phi\right)\right]$, où $\omega_{\mu}=\gamma_{\mu} H$ est la pulsation de la précession du spin dans le champ $H\left(\gamma_{\mu}=851.6 \mathrm{M} \operatorname{rad~s}^{-1}\right)$. La mesure de $\omega_{\mu}$ donne accés au champ $H$. La Figure 5 donne une représentation schématique d'un spectromètre $\mu \mathrm{SR}$ avec un seul "télescope à positrons" (dans la réalité il y en a plusieurs positionnés en anneau autour de l'axe du faisceau) et un aimant délivrant un champ appliqué vertical. On peut faire également des mesures en champ nul dans la phase d'ordre magnétique : le spin du muon précesse alors dans le champ dipolaire (et hyperfin) créé par les moments électroniques.

Une des caractéristiques de la spectroscopie de muons est d'être sensible à de très petits moments magnétiques ; en effet, on mesure couramment le flux de positrons jusqu'à des temps de $10 \mu$ s (quelques demi-vies du $\mu^{+}$), et un champ dipolaire de $5 \mathrm{G}$ génére une oscillation de période d'environ $15 \mu \mathrm{s}$. Des champs de quelques Gauss proviennent bien sûr des moments magnétiques nucléaires statiques, dont le physicien du solide se "débarrasse" en gelant leur contribution à la dépolarisation en appliquant un faible champ longitudinal, de quelques Gauss également. Ils peuvent provenir aussi de moments électroniques très faibles, de l'ordre de $0.01 \mu_{B}$, comme dans certains composés intermétalliques de terres rares ou d'actinides, ou bien dans des matériaux organiques [5, 6]. De telles valeurs de moment sont difficilement décelables par diffraction de neutrons, et la spectroscopie de muons montre alors tout son intérêt.

Par exemple, le composé organique $\mathrm{C}_{13} \mathrm{H}_{16} \mathrm{~N}_{3} \mathrm{O}_{4}$ (p-NPNN) posséde un électron non-apparié associé au groupe $\mathrm{N}-\mathrm{O}$, et le recouvrement des orbitales moléculaires rend possible le ferromagnétisme. Effectivement, des mesures macroscopiques ont mis en évidence dans p-NPNN une température de Curie très basse : $T_{C}=0.6 \mathrm{~K}$. On s'attend à ce que le moment magnétique spontané soit faible, du fait de la délocalisation de l'électron non-apparié. Des mesures $\mu \mathrm{SR}$ [5] dans ce composé ont décelé une dépolarisation oscillante dans le temps pour $T<T_{C}$; à $0.045 \mathrm{~K}$, le champ dipolaire responsable de cette oscillation vaut $150 \mathrm{G}$ (voir Fig. 6 à gauche). C'est une confirmation de la présence d'ordre magnétique dans p-NPNN, mais la grandeur du moment ne peut qu'être estimée car on ne connaît pas le site d'implantation du muon. À l'aide de la régle " $1 \mu_{B}$ à 1 Å donne un champ dipolaire de 1 kG", on peut penser que le moment magnétique associé au champ mesuré vaut 0.1-0.2 $\mu_{B}$. La régle précédente valant pour un moment localisé, le véritable moment magnétique spontané "délocalisé" est peut-être encore plus faible. À l'aide de ces spectres $\mu \mathrm{SR}$, on peut suivre assez précisément la variation thermique du champ dipolaire, et donc du moment, qui tombe bien à zéro vers $0.6 \mathrm{~K}$ (voir Fig. 6). Par diffraction de neutrons, les auteurs de la Ref. [7] ont réussi à observer une très faible augmentation de l'intensité des raies de Bragg nucléaires (1\%) au-dessous de $0.7 \mathrm{~K}$, ce qui démontre que la structure est ferromagnétique 

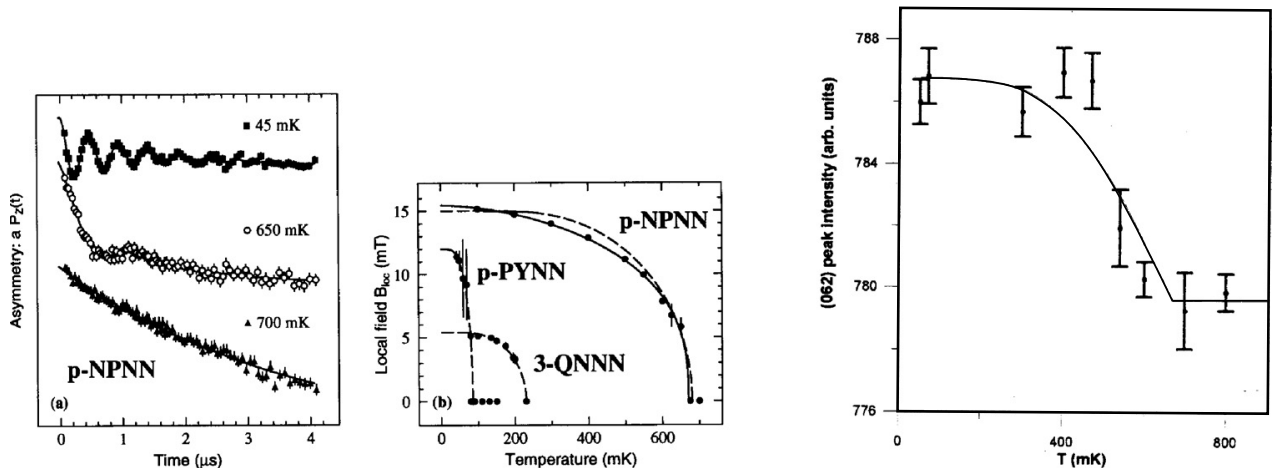

Figure 6. À gauche : dépolarisation du spin du $\mu^{+}$dans le ferromagnétique organique p-NPNN à différentes températures, et variation thermique du champ local mesuré sur les spectres (d'après les Refs. [5, 6]) ; À droite : variation thermique de l'intensité de la raie de Bragg (062) obtenue par diffraction de neutrons dans le même composé (d'après la Référence [7]).

avec $\mathbf{k}=\mathbf{0}$ (voir Fig. 6 à droite). Dans ce cas, l'expérience de diffraction de neutrons est difficile, car le signal magnétique est très faible, et les spectres $\mu$ SR fournissent une variation thermique du moment magnétique bien plus précise. Aucune des deux techniques, cependant, ne donne accès à la valeur du moment ordonné.

\section{LA RÉSONANCE MAGNÉTIQUE NUCLÉAIRE}

Le principe de la RMN est bien connu [8] : il s'agit de suivre l'absorption de puissance de l'ensemble des spins nucléaires d'un isotope donné, soumis à un champ magnétique externe, en fonction de divers paramétres, dont le champ appliqué et la température sont les principaux. La détection est de type électromagnétique, c'est-à-dire que l'on va mesurer la tension, induite aux bornes d'une bobine enroulée autour de l'échantillon, par les variations de l'aimantation nucléaire. Ces variations sont créées par un champ alternatif radiofréquence (de quelques dizaines de $\mathrm{MHz}$, variant suivant l'isotope) appliqué perpendiculairement au champ statique. Il n'est pas possible de donner ici un aperçu complet des différentes méthodes de spectrométrie RMN, qui se sont extraordinairement développées depuis sa découverte en 1946. Le lecteur intéressé est renvoyé aux Références [8,9]. Pour notre exposé, il suffit de savoir qu'une méthode courante consiste à envoyer sur l'échantillon de la puissance rf à une fréquence $v$ fixe, et à faire varier le champ magnétique appliqué $H_{0}$ : la résonance a lieu lorsque lorsque $h \nu=\gamma \hbar H$, où $\gamma$ est le rapport gyromagnétique du noyau considéré (dans son état fondamental) et $H$ le module du champ total (champ appliqué plus éventuellement champ interne) sur le noyau. On mesure alors la puissance absorbée en fonction du champ appliqué, ce qui donne la "forme de raie" RMN. Pour le proton $(I=1 / 2)$, qui reste le noyau le plus utilisé, la fréquence de résonance pour un champ appliqué de $1 \mathrm{~T}$ est $42.57 \mathrm{MHz}$.

Dans un matériau magnétique, on peut utiliser la résonance du noyau de l'atome magnétique lui-même, ou bien celle du proton (si le matériau en question en contient). L' avantage est que le signal du proton est très accessible en fréquence et peut être plus fort que celui des noyaux de l'atome magnétique. Dans la phase d'ordre magnétique, le proton est soumis à un champ dipolaire provenant des moments spontanés électroniques, qui va s'ajouter au champ appliqué et qui disparaît lors du passage en phase paramagnétique. La RMN est donc une technique capable de donner des informations dans le domaine du magnétisme électronique; elle est intéressante dans les composés contenant de l'hydrogène, qui absorbe fortement les neutrons, ce qui peut rendre les expériences de diffraction délicates. 

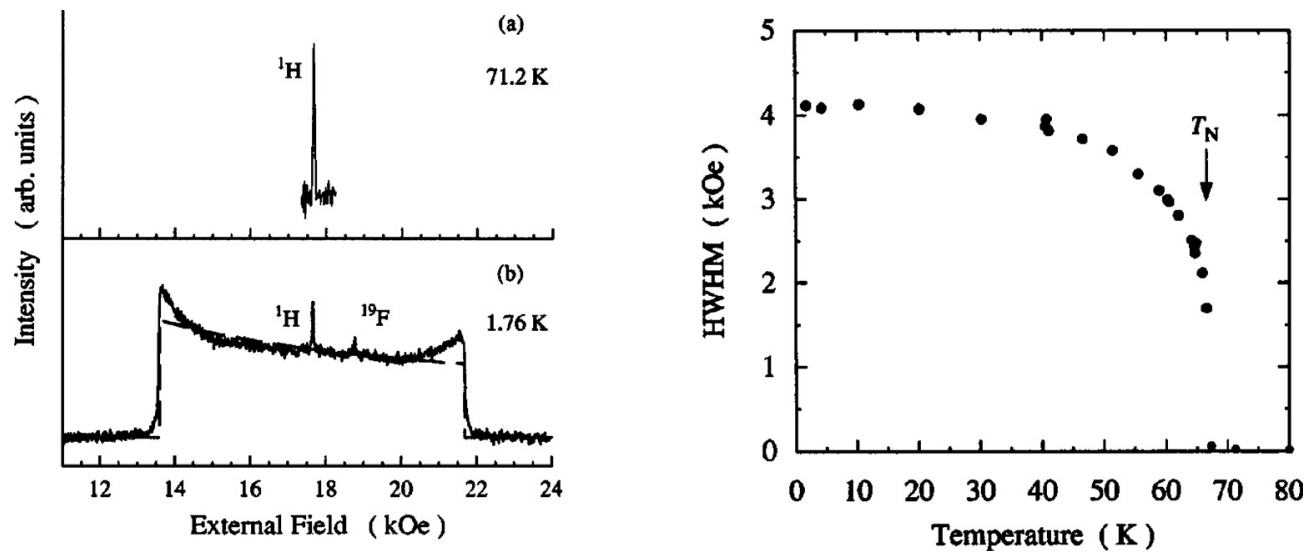

Figure 7. RMN sur ${ }^{1} \mathrm{H}$ dans la K-jarosite : À gauche : raie RMN à $71.2 \mathrm{~K}$ (en haut) et à $1.76 \mathrm{~K}$ (en bas). La fréquence rf est fixée à $75 \mathrm{MHz}$; À droite : variation thermique de la largeur de la raie RMN. Elle est proportionnelle au moment magnétique spontané du Fe $\mathrm{Fe}^{3+}$ (d'après la Ref. [10]).

En guise d'exemple, nous citerons l'étude par RMN sur ${ }^{1} \mathrm{H}$ de la jarosite de potassium $\mathrm{KFe}_{3}(\mathrm{OH})_{6}\left(\mathrm{SO}_{4}\right)_{2}$, qui contient les ions magnétiques $\mathrm{Fe}^{3+}$ dont les moments s'ordonnent au-dessous de $T_{N} \simeq 65 \mathrm{~K}[10]$. Dans ce type de composé, le probléme physique intéressant est la frustration géométrique des interactions antiferromagnétiques entre ions $\mathrm{Fe}^{3+}$ sur le réseau kagomé, formé de triangles se touchant uniquement par leurs sommets. La figure 7 à gauche présente la raie RMN sur ${ }^{1} \mathrm{H}$ dans la K-jarosite, en phase paramagnétique à $71.2 \mathrm{~K}$, et dans la phase d'ordre magnétique à $1.76 \mathrm{~K}$. La différence entre les deux raies est du même type que la différence entre les spectres Mössbauer en phase paramagnétique et en phase ordonnée : pour $T>T_{N}$, les noyaux des protons ne sont soumis qu'au champ appliqué, la raie de résonance est étroite et se trouve à $H_{r e s}=\omega / \gamma$. Dans la phase d'ordre magnétique, le champ total auquel est soumis un proton en un site donné vaut : $\mathbf{H}=\mathbf{H}_{0}+\mathbf{H}_{d i p}$, où $\mathbf{H}_{0}$ est le champ appliqué et $\mathbf{H}_{\text {dip }}$ le champ dipolaire provenant des moments magnétiques des ions $\mathrm{Fe}^{3+}$. Le module du champ total est donc : $H_{t}(\theta)=\sqrt{H_{d i p}^{2}+H_{0}^{2}+2 H_{d i p} H_{0} \cos \theta}$, si $\theta$ est l'angle entre le champ appliqué et le champ dipolaire. Lorsque l'échantillon est polycristallin, comme dans les mesures présentées ici, il existe une distribution d'angles $\theta$, et la résonance a lieu pour tout une gamme de champs appliqués $H_{0}$ tels que : $H_{\text {res }}-H_{\text {dip }}<H_{0}<H_{\text {res }}+H_{\text {dip }}$. La figure 7 à gauche illustre bien cette situation : la raie en phase paramagnétique $(T=71.2 \mathrm{~K})$ est très fine, alors que la raie à $1.76 \mathrm{~K}$, en phase ordonnée, est large et symétrique de part et d'autre de $H_{\text {res }} \simeq 17.5 \mathrm{kOe}$. On peut alors mesurer le champ dipolaire auquel est soumis le spin de ${ }^{1} \mathrm{H}$ de la part des moments ordonnés de $\mathrm{Fe}^{3+}$ à cette température : $H_{d i p} \simeq 4 \mathrm{kOe}$. Ce champ dipolaire étant proportionnel au moment spontané, on peut tracer sa variation thermique (Fig. 7 à droite), qui confirme la valeur de $T_{N} \simeq 65 \mathrm{~K}$ obtenue par des mesures antérieures de susceptibilité magnétique et diffraction de neutrons [11]. On peut remarquer que la structure magnétique de la K-jarosite a été résolue sur un échantillon deutéré, pour éliminer le fort bruit de fond dû à la présence d'hydrogéne dans le composé. La deutération est en principe neutre vis-à-vis des propriétés magnétiques, mais ce n'est pas toujours le cas; la RMN permet de faire l'étude sur le composé hydrogéné original.

\section{CONCLUSION}

Ces quelques exemples doivent montrer la véritable complémentarité des mesures par des techniques locales et par diffraction de neutrons dans le domaine du magnétisme statique, qui se vérifie également 
dans le domaine de la dynamique des moments, dont nous n'avons pas parlé. Les spectroscopies locales ne donnent en général pas d'informations détaillées sur la structure magnétique, mais :

- elles sont utiles dans les matériaux contenant des noyaux absorbant fortement les neutrons (Gd, Eu, $\mathrm{H}$... .).

- en cas d'ambigüité sur la structure, elles peuvent permettre de trancher entre différentes solutions.

- elles fournissent des informations particulières sur les champs hyperfins, les symétries locales, les comportements critiques, la nature de la transition ...

Enfin, il est clair que toutes les informations provenant des différentes techniques d'investigation doivent être prises en compte pour obtenir une compréhension plus fine des phènoménes étudiés, même si des contradictions apparaissent lors de la comparaison des résultats. Il est bien connu que la résolution des contradictions est source de progrès.

\section{References}

[1] Pour un exposé général sur la spectroscopie Mössbauer, voir : Ch. Janot, “L'effet Mössbauer et ses applications", Masson (1972).

[2] Bonville P., Hodges J. A., Hulliger F., Imbert P., Jéhanno G., Marimon da Cunha J. B. and Ott H. R., Hyperfine Interactions 40, 381 (1988).

[3] Dönni A., Fischer P., Furrer A. and Hulliger F., Solid St. Commun. 71, 365 (1989).

[4] Pour une référence générale sur la spectroscopie de muons, voir : "Muons and Pions in Materials Research", edited by J. Chappert and R.I. Grynszpan (North-Holland, 1984).

[5] Blundell S. J., Pattenden P. A., Pratt F. L., Valladares R. M., Sugano T. and Hayes W., Europhys. Lett. 31, 573 (1995).

[6] Dalmas de Réotier P. and Yaouanc A., J. Phys.: Condens. Matter 9, 9113 (1997).

[7] Zheludev A., Ressouche E., Schweizer J., Turek P., Wan M. and Wang H., Solid St. Commun. 90, 233 (1994).

[8] Abragam A., "Les principes du magnétisme nucléaire”, Presses Universitaires de France (1961).

[9] Slichter C. P., "Principles of magnetic resonance", $3^{\text {eme }}$ édition (Springer Verlag, 1990).

[10] Nishiyama M., Maegawa S., Inami T. and Oka Y., Phys. Rev. B 67, 224435 (2003).

[11] Inami T., Nishiyama M., Maegawa S. and Oka Y., Phys. Rev. B 61, 12181 (2000). 